\title{
Análise da competitividade da produção de Soja no sistema de plantio direto no Estado de Mato Grosso do Sul
}

\author{
Maria Isabel da Silva Azevedo Alvim* \\ Lourival Batista de Oliveira Júnior**
}

\begin{abstract}
Resumo - O setor agrícola nacional, em fase de reorganização e adequação aos novos padrões de produção e comercialização, enfrenta o desafio de crescer de modo competitivo e sustentável, para atender a demanda interna e conquistar e manter espaço no mercado externo, fornecendo produtos e processos de qualidade, com sustentabilidade e a preços competitivos. Para atender a nova demanda de uma agricultura mais equilibrada, ecológica e economicamente, é necessária a prática de uma produção coerente com o desenvolvimento sustentável. Assim sendo, o objetivo deste trabalho é analisar a lucratividade do sistema de plantio direto, um modo de produção mais sustentável do que o sistema de plantio convencional, no Estado de Mato Grosso do Sul, usando como metodologia a Matriz de Análise de Políticas (MAP), pelo fato de que a mesma permite que se faça uma comparação mais clara entre os dois sistemas. Pela análise dos resultados alcançados, percebe-se que o sistema de plantio direto apresentou custos mais baixos e maior lucratividade do que o sistema de plantio convencional, além de ser um sistema preservacionista, reduzindo as perdas de solo por erosão.
\end{abstract}

\footnotetext{
* Doutora em economia aplicada pela Universidade Federal de Viçosa/MG. isabel.alvim@ufjf.edu.br

** Mestre em políticas públicas e educação pela Universidade Federal de Juiz de Fora/ MG. lori@fea.ufjf.br
} 
Palavras-chave: desenvolvimento sustentável, competitividade, comercialização

\section{Classificação JEL: Q24}

Abstract: The Brazilian agricultural sector, which is undergoing the settlement of new production and trading patterns, faces the challenge of growing in a competitive and sustainable fashion, in order to attend domestic demand while acquiring and holding positions in foreign markets, and by providing quality products and processes with sustainability and competitive prices. To attend the new requirements form a more balanced agriculture, both in ecological and economic terms, it is necessary a practice of producing in a coherent fashion, with the sustainable development. Thus, the purpose of this article is to analyze the profitability of the direct planting system e production process which is more sustainable than the conventional system, in the State of Mato Grosso do Sul using the Policy Analysis Matrix (PAM) technique, which allows a more clear comparison between the two systems. On the basis of the results obtained, one can take note that the direct planting system displayed smaller costs and larger profitability than the conventional system, in additions to being a preserving system that reduces soil losses due to erosion.

Key-words: maintainable development, competitive, commercialization

JEL Classification: Q24

\section{I - Introdução}

A globalização recente da economia brasileira, com grande mobilidade de capital e transformações nas estruturas produtivas tradicionais, tem causado importantes modificações em diferentes setores. O país passou a fazer parte de um espaço integrado globalmente e bastante competitivo, provocando avanços tecnológicos e novos métodos de produção e de produtos, oriundos de cadeias produtivas integradas globalmente e onde a questão ambiental passa a ter importância fundamental nos processos 
de produção, comercialização e consumo. O crescimento acelerado de transações de bens que incorporam inovações tecnológicas e práticas ecologicamente corretas alteraram substancialmente as vantagens competitivas de vários segmentos econômicos em todos os países.

A maior parte das áreas agrícolas do Brasil são áreas sensíveis à erosão e de rápida degradação sob as ações do vento, chuva e sol quando cultivadas no sistema convencional. São solos que exigem alta tecnologia e oferecem condições para múltiplas culturas. Em algumas regiões são possíveis até três culturas por ano, devido ao clima. Mas quando o cultivo acontece no sistema convencional, a base produtiva se degrada em poucos anos.

$\mathrm{Na}$ análise econômica verifica-se que a erosão leva a um aumento dos custos de produção das culturas, requerendo gastos adicionais para reposição da fertilidade do solo, o que determina, dentre outras medidas, a adoção de técnicas de conservação que no médio prazo diminuam custos de produção.

O sistema de plantio direto (SPD) tem demonstrado sua eficácia na solução dos problemas de solo, primeiramente, com a introdução de práticas de cobertura de solo no inverno e a rotação de culturas, sendo que os testes feitos na cobertura de inverno comprovaram seus efeitos nas lavouras de verão. Avaliar os efeitos da eficácia e eficiência do SPD como contribuição decisiva para uma agricultura sustentável em termos ambientais, economicamente competitiva e socialmente eqüitativa tem sido o foco das atenções de pesquisadores e produtores, dentro do que se pode denominar "cadeia de sustentabilidade" da agricultura brasileira.

Neste contexto é objetivo deste trabalho analisar a competitividade e lucratividade da produção de soja no Estado de Mato Grosso do Sul, comparando-se os sistemas de plantio direto e plantio convencional (SPC). A escolha do estado decorre deste ter utilizado por mais tempo sistema de plantio convencional e por apresentar menores problemas de erosão de solo do que os demais estados produtores de soja na região do cerrado brasileiro. Nestas duas últimas décadas a produção de soja tem ocorrido, em sua maior parte, no sistema de plantio direto por ser este um sistema de maior preservação de solo do que o sistema convencional. 


\section{2 - Metodologia}

A análise desenvolvida neste trabalho teve como suporte teórico, os conceitos econômicos de lucratividade, custos sociais e privados de fatores, competitividade de sistemas de produção e política comercial. Os princípios analíticos desses conceitos baseiam-se na Teoria Neoclássica da Firma e Teoria do Comércio Internacional. O instrumental utilizado para essa análise foi a Matriz de Análise de Política (MAP), desenvolvida por MONKE \& PEARSON (1989).

CARDOSO \& BARROS (2002) ressaltam que não há na teoria econômica neoclássica uma definição para competitividade, sendo este um conceito político, ou seja, não há, na economia geral, uma teoria sobre a competitividade porque este não é um termo estritamente econômico. As transformações econômicas dos anos de 1980 e 1990 ampliaram a noção de competitividade das nações, estando superadas as visões econômicas tradicionais que definiam a competitividade como uma questão de preços, custos e taxas de câmbio. Uma definição particularmente influente afirma que competitividade para uma nação é o grau pelo qual ela pode, sob condições livres e justas de mercado, produzir bens e serviços que se submetam satisfatoriamente ao teste dos mercados internacionais enquanto, simultaneamente, mantenha e expanda a renda real de seus cidadãos (COUTINHO \& FERRAZ, 1995).

Geralmente, a competitividade é tida como sendo o resultado dos efeitos combinados de distorções de mercado e de vantagens comparativas. As distorções de mercado incluiriam tanto as causadas pela política econômica quanto pela competição imperfeita entre as firmas. Em conseqüência, se encontram na literatura os mais diferentes conceitos e indicadores para mensurar competitividade. A produtividade e a lucratividade são variáveis importantes na busca de competitividade.

Quando se fala em produtividade, é inerente pensar em rentabilidade, eficiência, tecnologia, inovação e condições de trabalho. A tecnologia compreende o estudo e a racionalização da produção, vinculados especialmente aos processos e métodos que transformam os insumos em produtos. O valor da tecnologia está relacionado com a aplicação que se faz dela para gerar riquezas ou melhorar a qualidade de vida.

A abordagem analítica deste trabalho baseou-se no modelo desen- 
volvido por MONKE \& PEARSON (1989) de estrutura organizacional denominado Matriz de Análise de Políticas (MAP), cuja atenção é dirigida para padrões eficientes de produção e preço e que permite obter uma avaliação dos efeitos de novas tecnologias sobre a lucratividade do sistema, através de comparações e variações nos orçamentos gerados pela alteração de uma subsérie de dados de insumos e de produção. Essas comparações proporcionam mais informações quanto à existência ou não de incentivos econômicos para promover a mudança tecnológica.

A MAP, conforme representação na Tabela 1, utiliza dois sistemas contábeis distintos em que se consideram respectivamente os preços de mercado (ou preços privados) e os preços sociais dos diferentes insumos e produtos. A estrutura da matriz permite estimar, com razoável grau de exatidão, os custos e os lucros da produção, revelando a maneira pela qual as políticas afetam a rentabilidade privada e a social de uma atividade. Os itens que compõem as planilhas são: insumos fixos, custo do trabalho, insumos intermediários, outros custos, custo total da produção agrícola, receita com produto e subproduto, lucro antes dos impostos, impostos diretos e lucro após o imposto.

Tabela 1 - Matriz de Análise de Políticas - MAP

\begin{tabular}{lcccc} 
Discriminação & Receita & Custos & & Lucro \\
& & $\begin{array}{c}\text { Insumos } \\
\text { Comercializáveis }\end{array}$ & $\begin{array}{c}\text { Fatores } \\
\text { Domésticos }\end{array}$ & \\
\hline Preços Privados & A & B & C & D \\
Preços Sociais & E & F & G & H \\
Efeitos de Divergências & & & & \\
e Eficiência Política & I & J & K & L \\
\hline
\end{tabular}

Fonte: MONKE \& PEARSON (1989).

Os cálculos da lucratividade privada obtida na primeira linha da Tabela 1 mostram a competitividade do sistema agrícola, das tecnologias atuais, os valores de produção, os custos dos insumos e as transferências.

A lucratividade é expressa da seguinte maneira:

$\mathrm{D}=\mathrm{A}-\mathrm{B}-\mathrm{C}$

Sendo: 
$\mathrm{A}=\mathrm{P}^{\mathrm{d}} \mathrm{Q}^{\mathrm{d}}$

Em que A é a receita privada, sendo $\mathrm{P}^{\mathrm{d}} \mathrm{o}$ preço do produto e $\mathrm{Q}^{\mathrm{d}}$ a quantidade total privada de determinado produto:

$$
\mathrm{B}=\sum_{\mathrm{i}=1}^{\mathrm{n}} \mathrm{p}_{\mathrm{i}}^{\mathrm{d}} \mathrm{q}_{\mathrm{i}}^{\mathrm{d}}
$$

Em que B éo custo dos insumos comercializáveis, $\mathrm{p}_{\mathrm{i}}^{\mathrm{d}}$ o preço privado do insumos i e $\mathrm{q}_{\mathrm{i}}^{\mathrm{d}}$ a quantidade privada do insumo $i$ utilizado do bem sendo analisado:

$$
C=\sum_{j=i}^{n} w_{j}^{d} l_{j}^{d}
$$

C é o custo dos insumos domésticos, em que $\mathbf{W}_{\mathrm{j}}^{\mathrm{d}}$ é o preço privado do insumo $\mathrm{j}$ e $\mathrm{j}_{\mathrm{j}}^{\mathrm{d}}$ quantidade privada do insumo $\mathrm{j}$ utilizado.

Os preços privados de mercado incorporam os efeitos de todas as políticas e imperfeições de mercado.

A primeira linha da matriz apresenta os cálculos da lucratividade privada (D) que indicam a competitividade do sistema de produção no período base, para determinado nível tecnológico, dados os valores dos produtos, os custos dos insumos e as políticas de transferências (como exemplo, impostos e subsídios) prevalecentes. Neste caso, o termo competitividade representa resultados financeiros na presença de efeitos de políticas e/ou imperfeições de mercado. Os resultados financeiros positivos (lucratividade) indicam que o sistema produtivo é competitivo dadas as condições existentes.

A segunda linha da matriz apresenta os valores sociais. Nesta linha, a lucratividade é calculada para avaliar a eficiência do sistema de produção agrícola. O conceito de vantagem comparativa é aplicado como medida de lucratividade social ou econômica, ou seja, a medida de vantagem comparativa indica a eficiência de alocação de recursos nacionais (ALVES, 2002). Portanto, a eficiência é obtida quando os recursos de uma economia são utilizados em atividades que proporcionam os maiores níveis de produção e renda. Desse modo, os lucros sociais (H) são uma medida de eficiência, desde que as receitas (E) e os custos de insumos (F + G) sejam avaliados em preços que refletem o custo de oportunidade social. O lucro social é dado por:

$\mathrm{H}=\mathrm{E}-\mathrm{F}-\mathrm{G}$ 
Sendo: $\mathrm{E}=\mathrm{P}^{\mathrm{s}} \mathrm{Q}^{\mathrm{s}}$

Em que E é a receita social, $\mathrm{P}^{\mathrm{s}}$ o preço social do produto e $\mathrm{Q}^{\mathrm{s}}$ a quantidade total do produto

$$
F=\sum_{i=1}^{n} p_{i}^{s} q_{i}^{s}
$$

Sendo F o custo dos insumos comercializáveis, $p_{i}^{s}$ o preço social do insumo i e $\mathbf{q}_{\mathrm{i}}^{\mathrm{s}}$ a quantidade do insumo utilizado;

$$
G=\left.\sum_{j=1}^{n} w_{j}^{s}\right|_{j} ^{s}
$$

Em que G é o custo dos insumos domésticos, $\mathbf{W}_{\mathbf{j}}^{\mathrm{S}}$ o preço social do insumo j e $\mathrm{I}_{\mathrm{j}}^{\mathrm{s}}$ a quantidade do insumo j utilizado.

Para produção (E) e insumos (F), que são comercializados mundialmente, considera-se que as avaliações sociais apropriadas são dadas pelos preços internacionais - preço de importação CIF para bens ou serviços que são importados ou preços de exportação FOB para os exportáveis. Considera-se que, a esses preços internacionais, os consumidores e produtores podem importar, exportar ou produzir bens e serviços domesticamente. $\mathrm{O}$ valor social da produção doméstica adicional compreende as reservas estrangeiras que não são dispendidas pela redução de importações, bem como o valor das reservas ganhas pela expansão das exportações (para cada unidade de produção, o preço de importação CIF ou de exportação FOB).

Como medida de eficiência ou vantagem comparativa, o lucro social $\mathrm{H}$ $=(\mathrm{E}-\mathrm{F}-\mathrm{G})$, quando negativo, indica que o sistema não é considerado economicamente viável no contexto de mercado internacional, sem assistência do governo, ou seja, tem-se uma indicação de que tal sistema não assegura a alocação economicamente eficiente de recursos escassos.

A terceira identidade, (I, J, K e L), refere-se às diferenças entre os valores privados e sociais de receitas, custos e lucros. Para cada entrada na matriz - mensurada verticalmente - uma eventual diferença entre o preço privado observado (mercado doméstico) e o preço social estimado (eficiência) deve ser atribuído aos efeitos de políticas (na forma de taxação, subsídios, restrições comerciais e distorções na taxa de câmbio) ou pela existência de falhas de mercados de produtos e de fatores. Essa relação é originada diretamente da definição de preço social (ALVES, 2002). 
Na tabela 2, pela análise da MAP expandida, cada entrada mede os efeitos de divergência e eficiência política (I, J, K e L) e é desagregada em três categorias:

Falhas de mercado (M, N, O, P);

Políticas distorcidas (Q, R, S, T); e

Políticas eficientes (U, V, W, X).

Tabela 2 - Expansão da Matriz de Análise de Políticas - MAP

Discriminação

Receita

Custos Lucro

Insumos

Comercializáveis

Fatores

Domésticos

\begin{tabular}{lllll}
\hline Preços Privados & A & B & C & D \\
Preços Sociais & E & F & G & H \\
Divergências e Efic. Política & I & J & K & L \\
Imperfeições de Mercado & M & N & O & P \\
Efeitos de Distorções Políticas & $\mathrm{Q}$ & $\mathrm{R}$ & $\mathrm{S}$ & $\mathrm{T}$ \\
Efeitos de Política Eficiente & $\mathrm{U}$ & $\mathrm{V}$ & $W$ & $X$ \\
\hline
\end{tabular}

Fonte: MONKE \& PEARSON (1989).

Esta tabela permite ainda se analisar outros dados tais como:

1 - Lucros Privados (LP): D = A - B - C

2 - Lucros sociais (LS): $\mathrm{H}=\mathrm{E}-\mathrm{F}-\mathrm{G}$

3 - Transferências associadas à produção: $\mathrm{I}=\mathrm{A}-\mathrm{E}=\mathrm{M}+\mathrm{Q}+\mathrm{U}$

4 - Transferências associadas ao custo insumos comercializáveis: $\mathrm{J}=$ $\mathrm{B}-\mathrm{F}=\mathrm{N}+\mathrm{R}+\mathrm{V}$

5 - Transferências associadas ao custo dos fatores: $\mathrm{K}=\mathrm{C}-\mathrm{G}=\mathrm{O}$ $+\mathrm{S}+\mathrm{W}$

6 - Transferências líquidas: $\mathrm{L}=\mathrm{D}-\mathrm{H}=\mathrm{I}-\mathrm{J}-\mathrm{K}=\mathrm{P}+\mathrm{T}+\mathrm{X}$.

A mensuração das transferências ${ }^{1}$ associadas à produção (I) podem ser representadas pela expressão:

$\mathrm{I}=\mathrm{A}-\mathrm{E}=\mathrm{M}+\mathrm{Q}+\mathrm{U}$

E as transferências associadas aos custos de insumos comercializáveis (J) são dados por:

$\mathrm{J}=\mathrm{B}-\mathrm{F}=\mathrm{N}+\mathrm{R}+\mathrm{V}$

${ }^{1}$ Considerando que as transferências decorrentes dos efeitos de políticas ocorrem no sentido dos produtores para os consumidores ou vice-versa. 
Considera-se que essas transferências resultam de dois tipos de políticas que causam divergência entre os preços domésticos dos produtos e os preços internacionais, caracterizadas como políticas específicas de produtos e política cambial.

Os custos sociais de fatores $(G)$ refletem condições de oferta e demanda subordinados aos mercados de fatores domésticos. Os preços de fator são, desse modo, influenciados pelo conjunto prevalecente de políticas macroeconômicas e de preço de produto. A atuação do governo pode ainda criar divergências entre custos privados (C) e custos sociais (G) por intermédio de política tributária ou de subsídios para um ou mais fatores de produção (capital, trabalho e terra).

As falhas de mercado, decorrentes de informações imperfeitas ou de desenvolvimento ineficiente das instituições que consistem em características do funcionamento de mercados, podem também influenciar os preços dos produtos e de fatores. Considerando que existem imperfeições de mercado de fator juntamente com políticas de fator "distorcivas", tem-se que ambos, $\mathrm{O}$ e $\mathrm{S}$, e possivelmente $\mathrm{W}$, são componentes positivos das transferências associadas ao custo dos fatores domésticos (K). Essas transferências são representadas como:

$$
\mathrm{K}=\mathrm{C}-\mathrm{G}=\mathrm{O}+\mathrm{S}+\mathrm{W}
$$

Dessa forma, a transferência líquida (L) combina efeitos de políticas "distorcivas" (I, J e S que são componentes de K) com aqueles de falhas de mercado de fator (representado por $\mathrm{O}$, componente de $\mathrm{K}$ ) e políticas eficientes para compensá-los (representado por W, componente de $\mathrm{K}$ ). A transferência líquida pode ser representada como:

$$
\mathrm{L}=\mathrm{D}-\mathrm{H}=\mathrm{P}+\mathrm{T}+\mathrm{X}
$$

Através de uma comparação de lucros privados e sociais, pode-se encontrar a transferência líquida. Essas medidas de transferência líquida devem, por definição, ser idênticas na matriz de contabilidade de dupla entrada, $\mathrm{L}=(\mathrm{I}-\mathrm{J}-\mathrm{K})=(\mathrm{D}-\mathrm{H})$. A desagregação da transferência líquida total indica que cada política "distorciva" fornece transferências positivas ou negativas para o sistema. Assim, a MAP permite a comparação dos efeitos de falhas de mercado e políticas "distorcivas" para o grupo inteiro de políticas de produtos e de "macropreço", assim considerados a taxa de câmbio e os preços de fatores, como a taxa de juros, salários e aluguel da terra. Mudanças nesses preços alteram os preços de insumos e produtos e dessa forma, influenciam a lucratividade agrícola. 
Os indicadores privados e sociais são obtidos das relações em que ambos os numeradores e denominadores de cada relação são extraídos da MAP em unidades de moeda nacional por unidade física do bem. Essas relações são as seguintes:

Razão do Custo Privado (RCP) calculada para proporcionar uma medida mais precisa de competitividade. Os resultados da lucratividade privada, que indicam a competitividade sob as condições de políticas existentes, são residuais e poderiam ter vindo de sistemas, utilizando níveis muito diferentes de insumos e de capital. Tal problema pode ser contornado pela construção de um indicador de custo privado (RCP), que é a razão entre os custos dos fatores domésticos a preços privados (C) e o valor adicionado a preços privados (A-B), isto é, $\mathrm{RCP}=\mathrm{C} /(\mathrm{A}-\mathrm{B})$.

Este indicador mostra quanto de renda o sistema de produção pode gerar para pagar os fatores domésticos e, ainda permanecer competitivo.

A razão do custo privado pode indicar ainda se os fatores domésticos estão recebendo o seu retorno normal $(\mathrm{RCP}=1)$, acima do retorno normal (RCP < 1) ou abaixo do retorno normal (RCP > 1) (ALVES, 2002).

Custo dos recursos domésticos (CRD) é uma medida de vantagem comparativa mais criteriosa que a lucratividade social; a razão é a mesma pela qual a RCP é mais precisa que a lucratividade privada, só que utilizando os valores sociais. Como indicador de vantagem comparativa (eficiência), o CRD indica o comportamento da lucratividade social, ou seja, quanto se despende de recursos domésticos em valores sociais para gerar uma unidade de divisas por meio de exportação, definido pela razão entre o valor social dos recursos domésticos (G) e o valor adicionado (E - F) expresso em equivalente social, isto é:

\section{$\mathrm{CRD}=\mathrm{G} /(\mathrm{E}-\mathrm{F})$}

Coeficiente de Proteção Nominal (CPN) indica o grau de transferência implícita no preço do produto, por exemplo, um CPN de 1,1 indica que prevalecem políticas que estão incrementando o preço de mercado em um nível $10 \%$ maior que o preço internacional. Interpreta-se o CPN como um indicador do grau de proteção ou desproteção (no sentido de que os efeitos das políticas são de beneficiar ou não os valores privados dos produtos e/ou insumos), dado aos sistemas produtivos. Quando esse coeficiente é menor do que 1 tem-se uma indicação de que os produtores 
do bem estariam sendo penalizados, à medida que recebem preços inferiores ao que pode ser considerado como nível de preço de eficiência.

Insumos comercializados internacionalmente estão sujeitos a tarifas, impostos ou subsídios. Nestas circunstâncias, o centro da análise deslocase da proteção ao produto final para a proteção que o sistema tributário concede ao processo produtivo. Define-se, então, a taxa de proteção efetiva como taxa de proteção ao valor adicionado em dado processo produtivo. Uma atividade qualquer só é verdadeiramente protegida se o resultado líquido da combinação da estrutura protecionista com os ajustamentos cambiais age no sentido de elevar o valor adicionado naquela atividade. $\mathrm{O}$ coeficiente de proteção efetiva consiste na razão entre o valor adicionado doméstico a preços de mercado e este valor a preços internacionais (ALVES, 2002).

Coeficiente de Proteção Efetiva (CPE) O coeficiente dessa razão mede o grau de transferências entre produtores e a sociedade derivada de políticas que afetam os mercados de produtos e insumos comercializáveis. Assim, é possível determinar quanto estas políticas afastam os preços dos produtos e dos insumos de seus preços de eficiência. Valores desse coeficiente menores do que 1 indicam que há desproteção ao produtor agrícola, uma vez que o valor adicionado na produção é inferior ao valor adicionado em termos econômicos. As transferências das políticas sobre o produto e sobre o insumo comercializável, tomadas em conjunto, são negativas sob a perspectiva do produtor que recebe e paga em valores privados. Os valores maiores que 1 indicam um resultado oposto, ou seja, os produtores são favorecidos.

Coeficiente de lucratividade (CL) é uma extensão do $\mathrm{CPE}$, que inclui as transferências entre os produtores e consumidores (sociedade) decorrentes dos efeitos de políticas sobre os fatores domésticos. O CL é a razão entre lucratividade privada e social, expresso como: $\mathrm{CL}=(\mathrm{A}$ $-\mathrm{B}-\mathrm{C}) /(\mathrm{E}-\mathrm{F}-\mathrm{G})=\mathrm{D} / \mathrm{H}$.

Razão de subsídios à produção (RSP) é o indicador final de incentivos. Final no sentido de ser conclusivo em termos de medida e também abranger os efeitos de políticas sobre toda a produção. É uma medida de transferência líquida de políticas (L) como uma proporção das receitas sociais totais (E). A expressão desse indicador assume a seguinte forma: $\mathrm{RSP}=\mathrm{L} / \mathrm{E}=(\mathrm{D}-\mathrm{H}) / \mathrm{E}$. 


\section{3 - Resultados e discussão}

São apresentados a seguir, os resultados da análise realizada para a produção de soja no Estado de Mato Grosso do Sul, empregando-se os sistemas de plantio direto (SPD) e plantio convencional (SPC), para as safras agrícolas de 2001/2002, 2003/2004 e 2004/2005, obtidos dos Comunicados Técnicos números 45, 77 e 90, fornecidos pela Embrapa Agropecuária Oeste, EMBRAPA/CPAO.

Todos os valores estão em reais ( $R$ \$) e referem-se a um hectare produzido. As variáveis analisadas são: os valores calculados para as lucratividades privada e social, os efeitos de divergências e eficiência de políticas, bem como os indicadores privados e sociais.

A Tabela 3 mostra a produção de soja na safra 2001/2002, em Mato Grosso do Sul, no sistema plantio direto.

Tabela 3 - Matriz de Análise de Política - MAP - produção de soja por hectare, safra 2001/2002 - MS, 2001. Sistema de Plantio Direto

\begin{tabular}{lcccc} 
Itens & Receitas & \multicolumn{2}{c}{ Custos } & Lucros \\
& & $\begin{array}{l}\text { Insumos } \\
\text { Comercializáveis }\end{array}$ & $\begin{array}{l}\text { Fatores } \\
\text { Domésticos }\end{array}$ & \\
\hline & $(\mathrm{A})$ & $(\mathrm{B})$ & $(\mathrm{C})$ & $(\mathrm{D})$ \\
Valores Privados & 1050,08 & 413,02 & 370,57 & 266,49 \\
& $(\mathrm{E})$ & $(\mathrm{F})$ & $(\mathrm{G})$ & $(\mathrm{H})$ \\
Valores Sociais & 1218,09 & 361,98 & 370,11 & 486,00 \\
Efeitos de Divergências & $(\mathrm{I})$ & $(\mathrm{J})$ & $(\mathrm{K})$ & $(\mathrm{L})$ \\
e Eficiência Política & $-168,01$ & 51,04 & 0,46 & $-219,51$ \\
\hline
\end{tabular}

Fonte: resultados obtidos pelos autores a partir de dados de EMBRAPA/CPAO (2002). 
A Tabela 4 traz os resultados da produção de soja no estado para o sistema de plantio convencional.

Tabela 4 - Matriz de Análise de Política - MAP - produção de soja por hectare, safra 2001/2002 - MS, 2001. Sistema de Plantio Convencional

\begin{tabular}{lcccc} 
Itens & Receitas & \multicolumn{2}{c}{ Custos } & Lucros \\
& & $\begin{array}{l}\text { Insumos } \\
\text { Comercializáveis }\end{array}$ & $\begin{array}{l}\text { Fatores } \\
\text { Domésticos }\end{array}$ \\
\hline & $(\mathrm{A})$ & $(\mathrm{B})$ & $(\mathrm{C})$ & $(\mathrm{D})$ \\
Valores Privados & 1050,08 & 415,70 & 415,50 & 218,88 \\
& $(\mathrm{E})$ & $(\mathrm{F})$ & $(\mathrm{G})$ & $(\mathrm{H})$ \\
Valores Sociais & 1218,09 & 367,75 & 404,44 & 445,90 \\
Efeitos de Divergências & $(\mathrm{I})$ & $(\mathrm{J})$ & $(\mathrm{K})$ & $(\mathrm{L})$ \\
e Eficiência Política & $-168,01$ & 47,95 & 11,06 & $-227,02$ \\
\hline
\end{tabular}

Fonte: resultados obtidos pelos autores a partir de dados de EMBRAPA/CPAO (2002).

A Tabela 5 mostra a produção de soja em Mato Grosso do Sul, na safra 2003/2004, utilizando o sistema de plantio convencional.

Tabela 5 - Matriz de Análise de Política, MAP, produção de soja por hectare, safra 2003/2004, MS, 2003 - Sistema de Plantio Convencional.

\begin{tabular}{lcccc} 
Itens & Receitas & \multicolumn{1}{c}{ Custos } & Lucros \\
& & $\begin{array}{c}\text { Insumos } \\
\text { Comercializáveis }\end{array}$ & $\begin{array}{l}\text { Fatores } \\
\text { Domésticos }\end{array}$ \\
\hline Valores Privados & 1618,20 & 487,55 & 645,46 & 485,19 \\
Valores Sociais & 1877,11 & 468,05 & 567,67 & 841,39 \\
Efeitos de divergências & & & & \\
e eficiência política & $-258,91$ & 19,50 & 77,79 & $-356,20$ \\
\hline
\end{tabular}

Fonte: resultados obtidos pelos autores a partir de dados de EMBRAPA/CPAO (2005a).

Na Tabela 6 abaixo, usando a MAP, obtem-se os valores da produção de soja, no mesmo estado pelo sistema de plantio direto para a safra $2003 / 2004$. 
Tabela 6 - Matriz de Análise de Políticas, MAP, produção de soja por hectare, safra 2003/2004, MS, 2003 - Sistema de Plantio Direto.

\begin{tabular}{lcccc} 
Itens & Receitas & $\begin{array}{l}\text { Custos } \\
\text { Insumos } \\
\text { Comercializáveis }\end{array}$ & $\begin{array}{l}\text { Fatores } \\
\text { Domésticos }\end{array}$ & Lucros \\
\hline Valores Privados & 1977,80 & 579,65 & 507,47 & 890,68 \\
Valores Sociais & 2294,25 & 556,46 & 468,09 & 1269,70 \\
$\begin{array}{l}\text { Efeito de Divergência. } \\
\text { e eficiência política }\end{array}$ & $-316,45$ & 23,19 & 39,38 & $-379,02$ \\
\hline
\end{tabular}

Fonte: resultados obtidos pelos autores a partir de dados de EMBRAPA/CPAO (2005a).

Pelas Tabelas 7 e 8 analisando-se a safra de 2004/2005 no estado selecionado também se pode verificar pelos resultados obtidos que a produção de soja continuou apresentando índices de lucratividade e competitividade tanto para o mercado interno quanto para o externo.

Tabela 7 - Matriz de Análise de Políticas, MAP, produção de soja por hectare, safra 2004/2005, MS, 2004 - Sistema de Plantio Convencional.

\begin{tabular}{lcccc} 
Itens & Receitas & \multicolumn{2}{c}{ Custos } & Lucros \\
& & $\begin{array}{l}\text { Insumos } \\
\text { Comercializáveis }\end{array}$ & $\begin{array}{l}\text { Fatores. } \\
\text { Domésticos }\end{array}$ & \\
\hline Valores Privados & 1861,00 & 799,38 & 775,67 & 285,95 \\
Valores Sociais & 2158,76 & 767,40 & 684,45 & 706,91 \\
$\begin{array}{l}\text { Efeitos de divergências } \\
\text { e eficiência política }\end{array}$ & $-297,76$ & 31,98 & 91,22 & $-420,96$ \\
\hline
\end{tabular}

Fonte: resultados obtidos pelos autores a partir de dados de EMBRAPA/CPAO (2005b).

Tabela 8 - Matriz de Análise de Políticas, MAP, Produção de soja por hectare, safra 2004/2005, MS, 2004 - Sistema de Plantio Direto.

Itens

Receitas

Custos

Lucros

Insumos Fatores

Comercializáveis Domésticos

\begin{tabular}{lcccc}
\hline Valores Privados & 2047,10 & 891,14 & 602,95 & 553,01 \\
$\begin{array}{l}\text { Valores Sociais } \\
\begin{array}{l}\text { Efeitos de Divergências e } \\
\text { eficiência política }\end{array}\end{array}$ & 2374,64 & 855,49 & 558,17 & 960,98 \\
\hline
\end{tabular}

Fonte: resultados obtidos pelos autores a partir de dados de EMBRAPA/CPAO (2005b). 
Pelos dados encontrados na matriz (MAP), passa-se a analisar os itens que respondem pela lucratividade e competitividade da produção de soja em Mato Grosso do Sul.

\section{1 - Lucratividade privada e social}

Os resultados apresentados sobre lucratividade, por hectare, indicam a competitividade dos sistemas analisados, do ponto de vista privado, por apresentarem lucros positivos. Indicam ainda, que a produção de soja, no Estado é competitiva e lucrativa, mesmo sob as condições atuais das políticas públicas.

As lucratividades sociais positivas indicam que há eficiência econômica nos dois sistemas e que há eficiência na geração de divisas e na alocação de recursos naturais nacionais

Os valores maiores do SPD mostram a eficiência e a maior competitividade deste, não só em termos de obtenção de menores custos de produção, quanto também na conservação e preservação dos recursos naturais, por ser um sistema já consagrado de preservação de solo.

\section{2 - Transferências associadas à produção}

As transferências associadas à produção de soja, nos SPD e SPC, apresentaram valores negativos para todas as safras, demonstrando que o produto, um dos principais produtos de exportação do Brasil, tem seu desempenho, de aumento ou retração da produção, atrelado ao desempenho do mercado mundial.

Comparando-se os preços, praticados no mercado de Mato Grosso do Sul, e os preços internacionais de soja, observa-se que há uma transferência negativa nos dois sistemas de produção, da ordem de aproximadamente $17 \%$. Isto significa que para cada hectare produzido, o produtor local, recebe menos do que ele poderia receber caso políticas de taxação à produção (ICMS e outros impostos) não tivessem sido implementadas. 


\section{3 - Transferências associadas aos custos de produção.}

Os cálculos associados aos custos dos insumos comercializáveis indicam que as transferências são positivas. Em relação aos insumos comercializáveis, os efeitos das divergências entre as avaliações sociais e privadas, são atribuídos às políticas distorcivas, e não às falhas de mercado. Essas políticas fazem com que os preços de mercado (privado) destes insumos, difiram dos preços mundiais (sociais).

A análise das transferências associadas aos custos dos insumos comercializáveis para a soja, indica que o governo tem adotado políticas intervencionistas no mercado deste produto, modificando as relações entre as avaliações privadas e sociais dos custos dos insumos comercializáveis, e a soja seria mais competitiva se fossem reduzidas ou mesmo eliminadas certas alíquotas de importação de insumos.

Os valores positivos representam custos privados maiores que os custos sociais dos fatores domésticos, mostrando transferência negativa à produção de soja, já que contribui para uma redução nos lucros privados.

Os valores apresentados mostraram melhor situação para o SPD.

\section{4 - Indicadores privados e sociais}

Os indicadores privados e sociais, demonstrados nas Tabelas 9, 10 e 11 a seguir, permitem comparações entre os sistemas de produção analisados, possibilitando uma melhor avaliação desses sistemas diante das limitações econômicas impostas e principalmente em relação à melhor alocação de recursos.

Todas as safras analisadas apresentaram resultados muito semelhantes em termos de confirmação da maior viabilidade do sistema de plantio direto em relação ao sistema de plantio convencional.

Outro fator que se pode verificar pela análise dos resultados é que a produção de soja é bastante competitiva e gera divisas ao país, ou seja, a alocação eficiente dos recursos confere competitividade e lucratividade à expansão desta atividade. 
Tabela 9 - Coeficientes da lucratividade dos SPD e SPC de produção de soja. MS, 2001

\begin{tabular}{|c|c|c|}
\hline Indicadores Privados e Sociais & SPD & SPC \\
\hline 1 - Razão do Custo Privado & & \\
\hline $\mathrm{RCP}=\mathrm{C} /(\mathrm{A}-\mathrm{B})$ & 0,58 & 0,65 \\
\hline 2 - Custo dos Recursos Domésticos & & \\
\hline $\begin{array}{l}\mathrm{CRD}=\mathrm{G} /(\mathrm{E}-\mathrm{F}) \\
3 \text { - Coeficiente de Proteção Nominal }\end{array}$ & 0,43 & 0,48 \\
\hline $\begin{array}{l}\mathrm{CPN}=\mathrm{A} / \mathrm{E} \\
4 \text { - Coeficiente de Proteção Efetiva }\end{array}$ & 0,86 & 0,86 \\
\hline $\begin{array}{l}\mathrm{CPE}=(\mathrm{A}-\mathrm{B}) /(\mathrm{E}-\mathrm{F}) \\
5 \text { - Coeficiente de Lucratividade }\end{array}$ & 0,74 & 0,75 \\
\hline $\begin{array}{l}\mathrm{CL}=\mathrm{D} / \mathrm{H} \\
6 \text { - Razão do Subsídio }\end{array}$ & 0,55 & 0,49 \\
\hline $\mathrm{RS}=\mathrm{L} / \mathrm{E}$ & $-0,18$ & $-0,19$ \\
\hline
\end{tabular}

Fonte: resultados obtidos pelos autores a partir de dados de EMBRAPA/CPAO (2002).

Tabela 10 - Coeficientes de lucratividade da produção de soja, SPD e SPC, MS, 2003

\begin{tabular}{lcc} 
Indicadores & SPC & SPD \\
\hline RCP & 0,57 & 0,36 \\
C/ (A-B) & & \\
CRD & 0,40 & 0,27 \\
G/ (E-F) & & \\
CPN & 0,86 & 0,86 \\
A / E & & \\
CPE & 0,80 & 0,80 \\
(A-B) / (E-F) & 0,58 & 0,70 \\
CL & & \\
D / H & $-0,19$ & $-0,16$ \\
RS & & \\
L / E &
\end{tabular}

Fonte: resultados obtidos pelos autores a partir de dados de EMBRAPA/CPAO (2005a).

Tabela 11 - Coeficientes de lucratividade da produção de soja - SPD e SPC, MS, 2004.

\begin{tabular}{lll}
\hline Indicadores & SPC & SPD \\
\hline RCP & 0,73 & 0,52 \\
CRD & 0,49 & 0,37 \\
CPN & 0,86 & 0,86 \\
CPE & 0,76 & 0,76 \\
CL & 0,40 & 0,57 \\
RS & $-0,19$ & $-0,17$ \\
\hline
\end{tabular}

Fonte: resultados obtidos pelos autores a partir de dados de EMBRAPA/CPAO (2005b). 
A razão do custo privado (RCP) é um indicador que mostra quanto o sistema produtivo pode produzir para pagar os fatores domésticos e, ainda, permanecer competitivo.

Os resultados apresentados, para o SPD e para o SPC, em preços privados menores do que 1, indicam que os fatores domésticos estão recebendo acima do retorno normal, ou seja, são lucrativos do ponto de vista econômico.

Mesmo ambos os sistemas sendo lucrativos, o SPD forneceu um indicador de custo privado menor do que o SPC, o que lhe confere maior potencial de expansão.

O custo do recurso doméstico (CRD) é calculado para avaliar o comportamento da lucratividade social dos sistemas e é analisado de forma análoga à da lucratividade privada, ou seja, minimizar o CRD é equivalente a maximizar os lucros sociais. Mede-se aqui a eficiência do sistema de produção ou sua vantagem competitiva.

Os resultados para SPD, e para SPC, foram inferiores à unidade, indicando que os valores sociais dos recursos domésticos empregados na produção de soja, são inferiores aos valores sociais adicionados. A utilização de recursos domésticos para gerar uma unidade monetária de divisas por meio de exportação é menor que a unidade, indicam que custos menores do que RS $\$ 1,00$, tanto para o SPD quanto para o SPC, em recursos domésticos, seriam suficientes para gerar US\$1,00 de divisas por meio da exportação. A expansão dessa atividade representa ganhos líquidos para o país, considerando-se, em termos de eficiência econômica, a alocação de recursos. O SPD, novamente apresentou maior eficiência econômica na alocação dos recursos.

O Coeficiente de Proteção Nominal (CPN) indicou que o produtor de soja em MS está recebendo menos que no mercado internacional. $\mathrm{O}$ coeficiente igual para os dois sistemas indica que o uso da tecnologia de produção, neste fator, não teve influência nos níveis de desproteção registrados.

O coeficiente de proteção efetiva (CPE), é um indicador que combina os efeitos dos indicadores anteriores a fim de verificar a extensão dos incentivos que os sistemas de produção recebem das políticas governamentais. O CPE estima o quanto as políticas que afetam o mercado de produtos fazem o valor adicionado diferir do valor que ocorreria na sua 
ausência. Os coeficientes aqui registrados indicam praticamente a mesma desproteção nos dois sistemas, aos fatores de produção.

O coeficiente de lucratividade (CL) é a razão entre o lucro privado e o lucro social e dimensiona a distância entre o lucro privado e o lucro que se obteria na ausência de políticas causadoras de distorção. Este coeficiente é considerado um indicador completo na proteção alcançada pelas políticas vigentes, pois leva em conta os efeitos de transferência de políticas no mercado de fatores medindo o efeito global de todas as políticas. Os valores dados os SPD e SPC indicam que a produção de soja está sendo liquidamente taxada, ou seja, indicam desproteção à atividade.

A razão de subsídio ao produtor (RS) é a transferência líquida de política como proporção da receita econômica total. Esta razão permite comparações sobre a extensão em que as políticas subsidiam os sistemas e pode ser desagregada para mostrar os efeitos de políticas de produtos, insumos e fatores. Os valores negativos apresentados em todas as safras e para os dois sistemas de produção demonstram que existe desproteção que provoca uma redução, mesmo pequena, na rentabilidade da produção de soja no estado.

\section{4 - Análise de sensibilidade}

A realização de uma análise de sensibilidade permitiu verificar como alterações na taxa de câmbio e nos fatores de conversão podem afetar a produtividade e competitividade da produção de soja no estado analisado, considerando-se que podem permanecer incertezas ou erros de estimação na metodologia utilizada.

Os impactos foram analisados sobre os indicadores de razão do custo privado (RCP), custo do recurso doméstico (CRD), coeficiente de proteção nominal (CPN), coeficiente de proteção efetiva (CPE), coeficiente de lucratividade (CL) e razão do subsídio (RS).

A variação na taxa de câmbio utilizada para os preços da soja, expressos em dólar, e dos insumos comercializáveis, foi de um incremento de $5 \%$ e de $10 \%$, mantendo os demais parâmetros constantes e iguais para os dois sistemas de produção.

A escolha de preços sociais para os produtos e insumos pode levar a uma imprecisão analítica. Isto porque, no processo de valoração social, os 
fatores de conversão utilizados podem apresentar falhas no seu processo de obtenção, por exemplo, a omissão de informações relevantes de impostos e tarifas. Nesse caso, uma mudança nos fatores de conversão dos valores privados para sociais, pode indicar os efeitos dessa variação sobre os indicadores da MAP. Para medir essa variação, aplicou-se aos indicadores da MAP uma variação de $5 \%$ e de $10 \%$ nos fatores de conversão.

São analisados também os efeitos que podem ocorrer nesses indicadores, com uma variação na taxa de câmbio.

\section{1 - Variação na taxa de câmbio}

A Tabela 12 mostra o resultado de variações de $5 \%$ e de $10 \%$ na taxa de câmbio nos indicadores da MAP.

Tabela 12 - Análise de sensibilidade dos indicadores da MAP dado um incremento de $5 \%$ e de $10 \%$ na taxa de câmbio(R $\$$ /US\$) para a produção de soja, MS-2001 - SPD e SPC

\begin{tabular}{lcccccr}
\hline Indicadores Privados e Sociais & \multicolumn{3}{c}{ SPD } & \multicolumn{3}{c}{ SPC } \\
\hline & Base & $5 \%$ & $10 \%$ & Base & $5 \%$ & $10 \%$ \\
\hline $1-\mathrm{RCP}=\mathrm{C} /(\mathrm{A}-\mathrm{B})$ & 0,58 & 0,58 & 0,58 & 0,65 & 0,65 & 0,65 \\
$2-\mathrm{CRD}=\mathrm{G} /(\mathrm{E}-\mathrm{F})$ & 0,43 & 0,41 & 0,40 & 0,48 & 0,45 & 0,44 \\
$3-\mathrm{CPN}=\mathrm{A} / \mathrm{E}$ & 0,86 & 0,82 & 0,79 & 0,86 & 0,82 & 0,79 \\
$4-\mathrm{CPE}=$ ( A - B ) / ( E - F ) & 0,74 & 0,71 & 0,69 & 0,75 & 0,71 & 0,69 \\
$5-\mathrm{CL}=\mathrm{D} / \mathrm{H}$ & 0,55 & 0,50 & 0,50 & 0,49 & 0,45 & 0,43 \\
$6-\mathrm{RS}=\mathrm{L} / \mathrm{E}$ & $-0,18$ & $-0,20$ & $-0,21$ & $-0,19$ & $-0,21$ & $-0,22$ \\
\hline
\end{tabular}

Fonte: resultados obtidos pelos autores a partir de dados de EMBRAPA/CPAO (2002).

Os resultados alcançados indicam pequenas alterações a um incremento de $5 \%$ na taxa de câmbio e uma maior alteração para $10 \%$, em todos os indicadores, com exceção do indicador de Razão do Custo Privado, como era de se esperar, uma vez que este indicador não sofre interferência dos preços sociais em seu cálculo.

Tais resultados não comprometem o caráter competitivo da produção de soja, sendo que os coeficientes que apresentaram maior sensibilidade são os que dependem, para sua composição, dos valores sociais, os 
quais têm, em seus cálculos, a taxa de câmbio como um dos principais componentes.

Verificou-se também, a maior competitividade e lucratividade do SPD, frente ao SPC.

\section{2 - Variação nos fatores de conversão}

Os efeitos de variação de $5 \%$ e de $10 \%$ nos fatores de conversão sobre os indicadores da MAP para os períodos analisados, estão apresentados nas Tabelas 13, 14 e 15 abaixo.

Tabela 13 - Análise de sensibilidade sobre os indicadores da MAP, de um incremento de $5 \%$ e de $10 \%$ nos fatores de conversão sobre a produção de soja, MS, 2001 - SPD e SPC.

\begin{tabular}{lrrrrrr} 
Indicadores Privados e Sociais & \multicolumn{3}{c}{ SPD } & \multicolumn{3}{c}{ SPC } \\
& Base & $5 \%$ & $10 \%$ & Base & $5 \%$ & $10 \%$ \\
\hline $1-\mathrm{RCP}=\mathrm{C} /(\mathrm{A}-\mathrm{B})$ & 0,58 & 0,58 & 0,58 & 0,65 & 0,65 & 0,65 \\
$2-\mathrm{CRD}=\mathrm{G} / \mathrm{E}-\mathrm{F})$ & 0,43 & 0,43 & 0,44 & 0,48 & 0,48 & 0,49 \\
$3-\mathrm{CPN}=\mathrm{A} / \mathrm{E}$ & 0,86 & 0,82 & 0,79 & 0,86 & 0,82 & 0,79 \\
$4-\mathrm{CPE}=$ ( A - B ) / ( E - F ) & 0,74 & 0,71 & 0,69 & 0,75 & 0,71 & 0,69 \\
$5-\mathrm{CL}=\mathrm{D} / \mathrm{H}$ & 0,55 & 0,52 & 0,52 & 0,49 & 0,46 & 0,47 \\
$6-\mathrm{RS}=\mathrm{L} / \mathrm{E}$ & $-0,18$ & $-0,18$ & $-0,19$ & $-0,19$ & $-0,19$ & $-0,20$ \\
\hline
\end{tabular}

Fonte: resultados obtidos pelos autores a partir de dados de EMBRAPA/CPAO (2002).

Tabela 14 - Análise de sensibilidade sobre os indicadores da MAP, de um incremento de $5 \%$ e de $10 \%$ nos fatores de conversão sobre a produção de soja, MS, 2003 - SPD e SPC.

\begin{tabular}{lcccccr} 
Indicadores Privados e Sociais & \multicolumn{3}{c}{ SPD } & \multicolumn{3}{c}{ SPC } \\
& Base & $5 \%$ & $10 \%$ & Base & $5 \%$ & $10 \%$ \\
\hline $1-\mathrm{RCP}=\mathrm{C} /(\mathrm{A}-\mathrm{B})$ & 0,36 & 0,38 & 0,38 & 0,57 & 0,56 & 0,56 \\
$2-\mathrm{CRD}=\mathrm{G} /(\mathrm{E}-\mathrm{F})$ & 0,48 & 0,47 & 0,46 & 0,43 & 0,48 & 0,49 \\
$3-\mathrm{CPN}=\mathrm{A} / \mathrm{E}$ & 0,86 & 0,84 & 0,80 & 0,86 & 0,82 & 0,79 \\
$4-\mathrm{CPE}=(\mathrm{A}-\mathrm{B}) /(\mathrm{E}-\mathrm{F})$ & 0,75 & 0,72 & 0,70 & 0,74 & 0,71 & 0,69 \\
$5-\mathrm{CL}=\mathrm{D} / \mathrm{H}$ & 0,49 & 0,52 & 0,52 & 0,55 & 0,54 & 0,57 \\
$6-\mathrm{RS}=\mathrm{L} / \mathrm{E}$ & $-0,18$ & $-0,18$ & $-0,19$ & $-0,19$ & $-0,19$ & $-0,20$ \\
\hline
\end{tabular}

Fonte: resultados obtidos pelos autores a partir de dados de EMBRAPA/CPAO (2005a). 
Tabela 15 - Análise de sensibilidade sobre os indicadores da MAP, de um incremento de $5 \%$ e de $10 \%$ nos fatores de conversão sobre a produção de soja, MS, 2004 - SPD e SPC.

\begin{tabular}{lcccccc}
\hline Indicadores Privados e Sociais & \multicolumn{3}{c}{ SPD } & & & SPC \\
& Base & $5 \%$ & $10 \%$ & Base & $5 \%$ & $10 \%$ \\
\hline $1-\mathrm{RCP}=\mathrm{C} /(\mathrm{A}-\mathrm{B})$ & 0,52 & 0,52 & 0,52 & 0,73 & 0,73 & 0,73 \\
$2-\mathrm{CRD}=\mathrm{G} /(\mathrm{E}-\mathrm{F})$ & 0,37 & 0,43 & 0,44 & 0,48 & 0,48 & 0,49 \\
$3-\mathrm{CPN}=\mathrm{A} / \mathrm{E}$ & 0,86 & 0,82 & 0,80 & 0,86 & 0,82 & 0,80 \\
$4-\mathrm{CPE}=$ ( A - B ) / ( E - F ) & 0,75 & 0,74 & 0,69 & 0,76 & 0,72 & 0,70 \\
$5-\mathrm{CL}=\mathrm{D} / \mathrm{H}$ & 0,57 & 0,55 & 0,55 & 0,40 & 0,41 & 0,42 \\
$6-\mathrm{RS}=\mathrm{L} / \mathrm{E}$ & $-0,17$ & $-0,18$ & $-0,19$ & $-0,19$ & $-0,19$ & $-0,20$ \\
\hline
\end{tabular}

Fonte: resultados obtidos pelos autores a partir de dados de EMBRAPA/CPAO (2005b).

Um incremento de $10 \%$ nos fatores de conversão causa uma alteração um pouco mais significativa do que de $5 \%$ em todos os indicadores, com exceção, novamente, do coeficiente da Razão do Custo Privado, por ser este indicador construído com preços internos, não sofrendo influência dos preços do mercado internacional. Estas variações ocorrem com maior intensidade nos indicadores de produção que sofram uma maior inflência dos preços externos no valor da receita social, a qual depende diretamente do fator de conversão.

Tais resultados, para ambos sistemas de produção, SPD e SPC, mostram que as políticas públicas, embora, por vezes tenham reduzido a receita privada em relação à social, não chegam a comprometer a competitividade da produção de soja na região, ou seja, mesmo ocorrendo falhas nas políticas públicas que implicam em aumentos nos custos de produção para o setor agrícola, fica clara a potencialidade deste setor para o crescimento da competitividade nacional.

\section{5 - Conclusão}

A soja brasileira possui competitividade tecnológica, o que significa afirmar que seus níveis de produtividade são comparáveis aos de seus concorrentes.

A abertura comercial e a recente desoneração da carga tributária sobre as exportações exerceram um papel fundamental no desempenho da sojicultura, embora essas vantagens estejam contrabalançadas pelo lado dos 
custos de produção que sugerem a existência de alguns pontos de estrangulamento na competitividade do produto, tais como gastos com fertilizantes, custos financeiros, custos de transportes e impostos domésticos.

A lucratividade calculada para os dois sistemas de produção, indicou a competitividade da produção de soja para ambas tecnologias, do ponto de vista privado, por apresentarem lucros positivos. O lucro maior dado pelo SPD permite confirmar a preferência por este sistema de produção dado sua maior rentabilidade. A lucratividade social positiva calculada constitui um indicador de que existem vantagens comparativas nos dois sistemas analisados. Isto significa que não há perdas na alocação de recursos, pois está havendo produção com custos sociais inferiores ao custo de importação. O SPD apresentou maiores vantagens comparativas, o que comprova que além de sua maior lucratividade, este sistema é o mais indicado para se desenvolver uma agricultura sustentável, por ser melhor conservador de recursos naturais.

A MAP permitiu identificar que ambos os sistemas, tanto de plantio direto quanto de plantio convencional, para a produção de soja, na região, são lucrativos e competitivos, no entanto, maiores ganhos, em termos de uso dos recursos disponíveis e de custos de produção podem ser obtidos na SPD. Com isso, esta análise pode subsidiar a tomada de decisão para o sistema de produção a ser utilizado pelo produtor, bem como que políticas deveriam ser adotadas pelo governo, para obter resultados mais eficazes quanto à produtividade e competitividade do setor agrícola, ou seja, a eficiência econômica ou vantagem comparativa dos sistemas agrícolas.

\section{6- Referências bibliográficas}

ALVES, J.M. - Competitividade e tendências da produção de manga para exportação do nordeste brasileiro. Piracicaba, Tese (Doutorado) - ESALQ/USP. Escola Superior de Agricultura Luiz de Queiroz/ Universidade de São Paulo, 2002.

CARDOSO, C. E. L.; BARROS, G.S.C. - A quase renda como indicador de competitividade em cadeias agroindustriais: uma proposta a ser implementada na cadeia de fécula de mandioca no Brasil. In: XL Congresso Brasileiro de Economia e Sociologia Rural - Eqüidade e eficiência na agricultura brasileira. Passo Fundo/RS. 28 a 31 de julho/02. 
COUTINHO, L.; FERRAZ, J.C. - Estudo da Competitividade da Indústria Brasileira. Campinas: Papirus/UNICAMP, 1995.

EMBRAPA/CPAO (EMBRAPA AGROPECUÁRIA OESTE). Comunicado Técnico 45. EMBRAPA, 2002.

EMBRAPA/CPAO (EMBRAPA AGROPECUÁRIA OESTE). Comunicado Técnico 77. EMBRAPA, 2005a.

EMBRAPA/CPAO (EMBRAPA AGROPECUÁRIA OESTE). Comunicado Técnico 90. EMBRAPA, 2005b.

MONKE, E \& PEARSON, S. R - Policy Analysis for Agricultural Development. Ithaca and London: Cornell University, 1989.

Recebido em março de 2004 e revisto em julho de 2005 